\title{
Les Mots : les échos du silence dans l'autobiographie de Jean-Paul Sartre
}

\section{Introduction}

La plupart des œuvres de l'écrivain Jean-Paul Sartre sont restées inachevées, à l'instar de son traité d'ontologie phénoménologique, L'être et le néant (1943) ; la trilogie romanesque, Les chemins de la liberté (1945) ; la biographie, L'idiot de la famille (1972). Son autobiographie, Les mots (1964), est, elle aussi, vouée à l'inachèvement. Différemment des biographies, les autobiographies désignent « un genre ouvert, interminable », d'après Philippe Lejeune (1980: 161).

Le concept d'ouverture est fondamental pour la compréhension du parcours de l'écriture sartrienne. D'un côté, il démontre le propos philosophique sartrien selon lequel tous les actes humains sont provisoires et susceptibles d'être modifiés dans une série ininterrompue de transcendances successives; de l'autre, il ouvre les frontières, ce qui permet à Sartre de constituer son projet basé sur la représentation d'un « parler infini ». En fait, peu d'auteurs ont développé d'une façon si exhaustive autant de manières discursives d'élaboration de la pensée - romans, drames, essais, nouvelles, traités, critiques philosophiques et politiques -, rien n’a échappé indemne à la loucherie privilégiée du regard de ce personnage médiatique par excellence.

Nous nous proposons dans cet essai d'évaluer comment s'opère le passage du silence à la parole (qui, chez Sartre, s'annonce aussi comme silence). Nous supposons que l'usage excessif de la parole - l'inflation de manifestations discursives sur la quasi-totalité des aspects existants dans son temps historique - implique un leurre, dans la mesure où il dénonce paradoxalement l'indisponibilité de l'auteur de se révéler devant « le regard d'autrui ", juge inexorable, conscience regardante, qui circonscrit la pleine liberté d'être des individus.

Pour ce faire, nous privilégierons Les mots (1964) comme corpus principal d'analyse, parce que dans l'autobiographie sartrienne la relation entre le silence et la parole

Deise Quintiliano - professeure associée de Lettres Françaises à l'Institut de Lettres Néolatines de l'Université de l'État de Rio de Janeiro. Adresse pour correspondance : Rua Carlos de Vasconcelos, nº 52/404 - Rio de Janeiro, Tijuca, Brésil, Cep: 20.521-050 ; e-mail: deisequintiliano@uol.com.br 
se pose de manière récurrente. Nous nous appuierons sur la méthodologie développée par Philippe Lejeune, notamment dans Le pacte autobiographique, jalonné par une approche sémiologique du texte littéraire.

\section{Le silence solipsiste}

Le roman autobiographique Les mots, publié en fragments tout le long de l'année 1963, dans le journal Les Temps Modernes, dirigé par Sartre, s'achève sur la promesse d'une suite dans un "testament politique » qui ne verrait jamais le jour. L'abandon de la suite du projet autobiograpique s'explique en partie par la perte progressive de la vision de l'écrivain, rapportée par Simone de Beauvoir, dans La cérémonie des adieux, biographie consacrée à son " compagnon de route »: " Au début de décembre [de 1973], il [Sartre] n'allait pas plus mal ; mais il n'allait pas mieux : il dormait. Il dormait même le matin quand Victor lui faisait la lecture. C'était une fuite, j'en suis certaine : il ne pouvait pas accepter sa quasi cécité » (Beauvoir, 1982 : 90-92).

Sartre avait d'ailleurs déclaré que l'écriture de son autobiographie signifiait un adieu à la littérature :

Je voulais que ce soit agaçant, ce livre, je voulais que ça soit un adieu à la littérature qui se fasse en bel écrit. C’est-à-dire, je voulais que les gens qui lisent ça se trouvent entraînés dans une espèce de contestation de la littérature par elle-même, voilà (Astruc \& Contat, $1977: 54)$.

Cette déclaration confirme et explique le silence dans lequel plonge sa production littéraire postérieure. Faisant partie de son quotidien, la symbolique du silence représente, en outre, une menace qui guette sournoisement l'auteur. Dans un épisode concernant le douloureux et prolongé affrontement des problèmes avec ses dents, Simone de Beauvoir s'en souvient :

Sartre avait de nouveau des ennuis avec ses dents. Le dentiste lui dit qu'en octobre [de 1972] il faudrait lui poser un vrai râtelier et qu'il serait gêné pour parler en public. S'il ne pouvait plus parler dans des meetings ni même dans des réunions un peu nombreuses, il serait obligé de prendre sa retraite politique (Beauvoir, $1981: 51$ ).

Le projet d'un testament politique est alors remplacé par une série de témoignages, de films ou d'entretiens enregistrés dans des interviews accordées à Simone de Beauvoir et aux amis les plus proches, dans l'élaboration d'un ouvrage qui serait nommé plus tard « l'autobiographie parlée de Sartre ». Tous ces événements confondus dessinent un trajet qui mène du " parler absolu » au « taire absolu » du chef de file de l'existentialisme - mouvement déclenché par Sartre et ses acolytes, aux alentours de Saint-Germaindes-Prés, dans l'immédiat après-guerre, avant qu'il ne gagne le monde. 
Toutefois, le passage du silence à la parole - ou de la parole au silence, car les deux termes sont plutôt équivalents qu'antinomiques chez Sartre - ne se présente pas de façon linéaire, droite ou rectiligne, étant marqué par l'ambiguité et l'opacité. Cette ambiguïté est sous-jacente à la grande question que l'ensemble de l'œuvre philosophique et littéraire de Sartre met en lumière : la problématisation du sujet. C'est pourquoi, dans L'être et le néant, Sartre avait déjà essayé de répondre au questionnement fondamental que posent Les mots : «Qu'est-ce que le soi ? le soi renvoie au sujet, il n'est pas une plénitude d'existence, il implique un décollement de l'être par rapport à soi » (Sartre, $1943: 115)$.

La problématisation du sujet s'insère par ailleurs dans l'autobiographie sartrienne tout en introduisant l'idée d'ipséité et en soulignant le récit du "petit Poulou », surnom de l'enfant Sartre, dans un monde où il s'imposait d'emblée comme une plénitude : «l'idée ne me vint pas qu'on pût écrire pour être lu » (Sartre, $1964: 147$ ). Lorsqu'il admet, dans un premier temps, la possibilité de l'exercice scriptural au détriment de sa réalisation dialectique - intimement associée au processus de lecture - Sartre semble vouloir s'éclipser au regard du lecteur. Aussi, dans Les mots, les différentes étapes de la vie de l'auteur sont-elles enchevêtrées, superposées, fusionnées, ayant pour but de flouer le lecteur, de se replier sur elles-mêmes, de se taire même quand elles ont l'air de parler.

\section{Le dire dialectique}

L'une des caractéristiques qui singularisent Les mots en tant que construction littéraire est le fait que le texte s'avère être une métaphore autobiographique très convaincante qui cache sa profondeur. La division du roman en deux parties : lire et écrire n'est pas moins trompeuse. Le second terme présupposant le premier nous induit à l'erreur de considérer qu'une chronologie historique serait à la base des épisodes énoncés par le narrateur.

Certes, la plupart des autobiographies respectent « l’ordre chronologique » des événements, de la naissance jusqu'à la mort, comme si l'on vivait dans un feuilleton historique. Notre constitution en tant qu'individus s'articule dans une relation étroite avec l'altérité ; il est donc normal que la chronologie ait de l'importance dans un récit autobiographique qui signale notre inscription singulière dans l'histoire. Toutefois, Philippe Lejeune (1975: 204) nous avertit que, chez Sartre, où il semble avoir succession chronologique il y a, en réalité, simultanéité. L’organisation des épisodes est dialectique, c'està-dire le développement des événements mentionnés, décrits ou rappelés, correspond à la résolution progressive d'une série de crises qui s'originent les unes des autres.

Le passage de l'ipséité vers l'altérité s'établit par l'introduction de l'autre, de l’alter, du lecteur dans un enjeu dialectique, par l'intermédiaire duquel le " je " se définit comme sujet. Ainsi, dans la création de son système philosophique, en oubliant les chimères de Poulou, le Sartre adulte finirait par admettre : "On ne peut écrire sans 
public et sans mythe - sans un certain public que les circonstances historiques ont fait, sans un certain mythe de la littérature qui dépend, en une très large mesure, des demandes de ce public » (Sartre, $1995: 154)$.

Le philosophe met en valeur la fonction communicative de la littérature ; l'espace dialogique, l'interlocution, en superposant la parole au silence et en introduisant une cassure dans la structure monolithique du solipsisme. La façon dont Sartre se jette et se dérobe simultanément dans le jeu dialectique qui reste implicite dans Les mots, faisant ressortir le personnage du lecteur, amène Lejeune à reconnaître l'originalité du projet sartrien :

Seuls, parmi les modernes, Michel Leiris et Jean-Paul Sartre se sont trouvés en situation d'inventer de nouvelles structures de récit, parce qu'ils étaient sans doute les seuls, non seulement à saisir que le récit biographique n’allait pas de soi, mais à avoir réfléchi suffisamment qu'un renouvellement du récit autobiographique impliquait un renouvellement général de l'anthropologie, et des modèles de description et d'explication de l'homme (Lejeune, $1975: 202$ ).

En effet, Sartre est incontestablement le premier écrivain qui fonde la technique biographique basée sur l'adoption d'une méthode vraiment originale, déguisée sous la forme d'un livre classique des réminiscences d'enfance. Il ne s'agit pas d'appliquer une théorie générale à sa propre autobiographie, mais de modifier cette théorie en fonction de l'application et de la vérification des résultats.

Dévoilant les coulisses d'un discours où l'ordre logique se cache sous un ordre chronologique forgé, Philippe Lejeune (1975 : 204-224) montre comment le « dire autobiographique » masque un " taire absolu », dans la stratégie proposée par Sartre. Cette stratégie attire le lecteur dans d'innombrables pièges tendus par la ruse de l'écrivain.

\section{L'utopie de la transparence}

Une étude d'Alain Buisine, consacrée à l'analyse des fantasmes sartriens d'exhibition et de transparence, décèle l'image d'un Sartre « en perpétuelle reconstruction par ses propres soins, constamment en réfection, en restauration, et cependant jamais achevé, jamais véritablement établi » (Buisine, 1986 : 25). Elle nous montre Sartre comme un sujet : « toujours en manque de sa propre identité » et pour cela « de plus en plus dominé par une incontrôlable pulsion autobiographique, logorrhée impossible à endiguer, au point qu'il invente et perfectionne d'interview en interview un nouveau genre littéraire " (Buisine, $1986: 25$ ). C'est la manière par laquelle se dénude finalement le rêve sartrien d'exorciser « un fond sombre qui refuse d'être dit » (Sartre, $1976: 143$ ).

Dans ses Carnets de 1939-1940, Sartre avait déjà annoncé son désir de faire régner dans son esprit une "clarté impitoyable ». Septuagénaire, le même fantasme le hante : "J'estime que chacun devrait pouvoir dire, devant l'interviewer, le plus 
profond de soi. [...] j'essaie d'être le plus translucide possible. [...] j'essaie d'être le plus clair et le plus vrai possible, de manière à livrer entièrement, ou à essayer de livrer entièrement, ma subjectivité » (Sartre, $1976: 141-143$ ).

Ce mouvement vers la révélation, qui rompt le silence et instaure la parole, passe par la démystification des impostures et des mensonges qui marquent l'enfance du héros cabotin. Bien entendu, cette prise de position n'est pas exempte de critiques :

Qu'est-ce qui pousse donc un écrivain célèbre, déjà si reconnu que cela n’ajoutera vraiment rien à sa gloire, à ainsi prostituer sa biographie et son intimité, à s'exposer aussi dangereusement et dérisoirement sur la place publique ? Qu'est-ce que lui impose ce contrat exorbitant et indécent, tout dire, tout avouer, ne pas cesser de se confesser ? (Buisine, $1986: 28$ ).

Voici le rôle essentiel susceptible d'être assigné au roman autobiographique sartrien : celui d'hygiéniser les monuments sur lesquels toute la culture occidentale a été bâtie ; culture dans laquelle son historicité l'insère comme personnage en même temps fictif et historique.

Le roman établit ainsi un itinéraire de conversion du narrateur à l'authenticité, moyennant un éventail de rôles bien esquissés, joués par le petit Poulou tout au long du récit. Ce rite de conversion s'élabore de façon ambigüe, dans Les mots, inscrivant le récit dans une tension éternelle, provoquée par le heurt constant entre le silence et la parole.

\section{Les mots : des paroles qui se taisent}

Dans l'article "L'ordre du récit dans Les mots de Sartre », Philippe Lejeune (1975 : 197-243) analyse la façon dont Sartre, sous l'aspect d'un livre classique de souvenirs d'enfance, renouvelle la technique de l'écriture autobiographique, tout en remplaçant le développement chronologique par le mouvement dialectique. Nous présenterons, de manière succincte, l'essentiel des formulations de Lejeune, fort schématiques.

D’entrée de jeu, nous remarquons la présence de deux coupures chronologiques, dans la conclusion du livre Les mots : 1917 (date du remariage d'Anne-Marie, mère de Sartre) et 1940 (date du début de la rédaction de L'être et le néant). Dans le deuxième cas, grâce à un anachronisme spectaculaire, la vision de Poulou anticipe une réalisation future, qui n'aurait lieu que lors de la publication de Lềtre et le néant : "plus tard, j’exposai gaîment que l'homme est impossible »(Sartre, 1964 : 204).

L'ordre chronologique, à proprement parler, n'est respecté que dans l'introduction et la conclusion du roman. Dans l'espace intermédiaire : "Tous les événements ou sentiments sont traités comme s'ils étaient contemporains » (Lejeune, 1975 : 207). La plupart des faits racontés dans la première partie - lire - se passent entre 1909 et 1914, alors que ceux qui concernent la seconde - écrire - se superposent, étant légèrement décalés à l'égard des précédents, de 1912 à 1917. La technique adoptée 
confond le lecteur qui croit, à tort, accompagner le développement chronologique de l'histoire, ce qui autorise Lejeune (1975: 209) à affirmer : « L'ordre général du récit n'est donc pas celui d'une histoire. Il est celui d'une fable dialectique ».

Tout en dissolvant la division du livre en lire et écrire, qu'il juge artificielle, Lejeune dénonce la prestidigitation de l'écriture sartrienne, qui se replit toujours sur elle-même, dans une manœuvre qui instaure le silence, même lorsque l'auteur avoue son intention de se montrer tout entier dans sa subjectivité : "L'écrivain est à la fois quelqu'un qui se déguise et qui aspire à la transparence " (Idt, 1976: 75).

Dépassant les limitations interprétatives d'une lecture éminemment synchronique du texte littéraire, la structure du texte Les mots s'avère être celle d'un récit mythique dont les articulations dialectiques, selon Lejeune (1975:232), devront être représentées comme des schèmes de succession historique, travestissant l'ordre chronologique, voire inversant le cours des événements. C'est pourquoi, lorsque la comédie n'est pas satisfaisante, l'enfant y renonce tout court ; en revanche, si elle lui plaît, comme la joie que ressent Poulou vis-à-vis de la découverte de l'écriture clandestine, l'auteur invente une raison arbitraire pour que le récit puisse se poursuivre.

Tous les subterfuges, mis en œuvre par Sartre, inaugurent une nouvelle méthodologie d'analyse des faits autobiographiques. Comme l'affirme Lejeune (1998: 170), le programme que Sartre donne dans ses interviews de l'époque est ambitieux. Il va s'appliquer à lui-même - comme il ferait plus loin à Flaubert - des méthodes psychanalytiques et marxistes, ayant pour but d'examiner son histoire pour pouvoir tracer celle de sa génération tout entière.

Et pourtant, notre hypothèse est que l'exposition outrancière du personnage, partageant un " parler absolu » de ses aventures et mésaventures familiales, n'est qu'un repli sur soi-même. En d'autres mots, c'est une sorte d'irréductibilité discursive fondée sur un silence absolu, ce qui fait que Sartre revient au solipsisme primaire qui donnait tant de plaisir au "petit Poulou».

L'un des passages les plus significatifs du roman met l'accent sur cette clé de lecture, en l'occurrence, l'expérience de la laideur.

\section{L'expérience de la laideur}

L'histoire romancée de Sartre s'annonce comme un outil consistant et pertinent de vérification pragmatique de sa philosophie. L'enfance de l'auteur engendre des lois qui rassemblent tous les éléments, grâce auxquels l'écrivain élaborerait sa philosophie existentialiste. Pour Jacqueline Villani $(1996: 25)$, «sa propre enfance apparait donc comme une démonstration de la validité du modèle existentialiste, une illustration de ses thèses ». Aussi, Sartre passe-t-il son enfance en revue de manière tendancieuse et sélective, notamment lorsqu'il évalue la naissance de sa vocation d'écrivain.

Nonobstant cette hypothèse interprétative, c'est dans l'enfance portraiturée dans Les mots, que Sartre fait la découverte fatale de sa laideur physique : 
Un jour - j'avais sept ans - mon grand-père [...] me prit par la main, annonçant qu'il m'emmenait en promenade. Mais, à peine avions-nous tourné le coin de la rue, il me poussa chez le coiffeur en me disant : "nous allons faire une surprise à ta mère." [...] je regardai avec bienveillance mes boucles couler le long de la serviette blanche qui me serrait le cou et tomber sur le plancher [...] je revins glorieux et tondu. Il y eut des cris mais pas d'embrassements et ma mère s'enferma dans sa chambre pour pleurer : on avait troqué sa fillette contre un garçonnet. Il y avait pis : tant qu'elles voltigeaient autour de mes oreilles, mes belles anglaises lui avaient permis de refuser l'évidence de ma laideur. Déjà, pourtant, mon œil entrait dans le crépuscule. [...] on lui avait confié sa petite merveille, il avait rendu un crapaud. [...] Anne-Marie eut la bonté de me cacher la cause de son chagrin. Je ne l'appris qu’à douze ans, brutalement (Sartre, 1964 : 86-87).

Après avoir constaté sa laideur d'une façon traumatique, le premier mouvement que le héros esquisse est celui de l'esquive dans le but de s'échapper de cette inexorable évidence, tout en se cachant derrière des " grimaces " ou des "singeries ", très bien articulées par le comédien Poulou:

Quand je me les rappelle aujourd'hui, ces grimaces, je comprends qu'elles assuraient ma protection : contre les fulgurantes décharges de la honte, je me défendais par un blocage musculaire. Et puis, en portant à l'extrême mon infortune, elles m’en délivraient : je me précipitais dans l'humilité pour esquiver l'humiliation [...] le miroir m’était d'un grand secours : je le chargeais de m’apprendre que j’étais un monstre (Sartre, 1964 : 90-91).

Ce passage renforce notre sentiment selon lequel, lorsque Sartre assume l'activité littéraire, il continue de jouer sa comédie enfantine, maintenant déjà inséré dans les canons des adultes - devenant écrivain - cherchant à se dérober du " regard d'autrui ». Comme lui-même nous avertit dans Les mots, il est toujours un pas avant cet " autrui », juge implacable qui l'examine et le pétrifie dans son " en-soi », dans sa laideur - élément majeur dans l'acheminement de Sartre vers les mots silencieux - qui, par l'intermédiaire d'un truquage, l'écrivain cherche à tout prix dissimuler.

Cependant, si Sartre devient écrivain pour attirer l'attention sur lui-même, ayant pour but d'entraîner le mouvement inverse - détournant les réflecteurs pour se faire invisible - cette manœuvre n'est possible que par le truchement de la ruse et du leurre, qui sont des éléments récurrents dans son récit autobiographique.

Selon cette hypothèse, Les mots se révèlent un fils du silence, (sous le déguisement du parler absolu), par l'inachèvement, par la comédie, par la dissimulation. Cette approche est renforcée par Philippe Lejeune qui dénonce l'existence d'un modèle complexe camouflé sous la linéarité aparente du récit.

La caractéristique la plus importante de l'autobiographie sartrienne peut être résumée par l'aphorisme suivant : «Enfant imaginaire, je me défendis par l'imagination (Sartre, 1964 : 94). En fait, c'est justement le travail formulé à partir de l'imagination qui permettra à l'auteur de pénétrer, par la suite, dans les chemins labyrinthiques de la fiction. 


\section{La Victoire du Silence}

Tout ce dont Sartre se souvient de son enfance, ce sont des remémorations ponctuelles, des séquences discontinues, des passages fragmentés transformés par son musée imaginaire en tissu fictif : «Fautes d'ennemis visibles, la bourgeoisie prenait plaisir à s'effrayer de son ombre [...]. On parlait de spiritisme, d'ectoplasme ; rue Le Goff, au numéro 2, face à notre immeuble, on faisait tourner les tables » (Sartre, $1964: 123)$.

Ces souvenirs se présentent comme des microrécits insérés de manière articulée, dans la trame dialectique sartrienne. Et pourtant, si les épisodes sont détachés de l'éventail de références intratextuelles qui les soutiennent, ils s'annoncent, comme l'affirme Villani (1996 : 75), "étrangement significatifs de tout un non-dit, notamment d'angoisses que l'analyse sartrienne tend à masquer, à détourner sur d'autres objets ».

Écriture qui ausculte son propre silence, Les mots réfléchissent également un lien fondamental de l'auteur avec son passé et sa famille. Elle annonce par ailleurs que la force du désir de destruction de ce lien est également proportionnelle à la profondeur même de cet attachement : "Détaché de son contexte, le portrait de 'l’enfant merveilleux' ne contient aucune trace d'ironie» (Villani, 1996:76).

La relation de Sartre avec le plus grand silence qui s'imposerait à lui, symbolisé par la cécité qui le frapperait des années plus tard, est aussi ironiquement vaticinée par le narrateur, moyennant des signes perçus dans son enfance troublée :

Anne Marie me trouvait à mon pupitre, gribouillant, elle disait : "Comme il fait sombre! Mon petit chéri se crève les yeux." C'était l'occasion de répondre en toute innocence : "Même dans le noir je pourrais écrire." Elle riait, m’appelait petit sot, donnait de la lumière [...] Je venais d'informer l'an trois mille de ma future infirmité. En effet, sur la fin de ma vie, plus aveugle encore que Beethoven ne fut sourd, je confectionnais à tâtons mon dernier ouvrage [...] qui, naturellement, serait mon chef-d’ouvre (Sartre, $1964: 167)$.

Aussi, montrant que l'écrivain - toujours ancré dans son temps historique, dans ses convictions politiques, dans sa formation culturelle - est un comédien à la fois manipulé et manipulateur, Sartre ouvre-t-il des possibilités d'interprétations subjectives des faits littéraires qu'il finit par représenter dans le rôle de personnage.

Tout en se dissolvant dans son propre discours, Les mots sartriens se taisent lorqu'ils atteignent la limite du dire excessif. En ce moment, il y a effectivement une conversion qui mène du silence solipsiste de Poulou à la dialectique silencieuse de Sartre, car le philosophe se montre textuellement pour se cacher physiquement.

Ainsi, comme le plus significatif d'un vase est le vide à l'intérieur, c'est en écrivant que Sartre s'engage dans une absence. En d'autres termes, il écrit de façon excessive dans l'essai de vérifier quel sentiment son absence serait à même de susciter après sa mort et pour que la postérité puisse s'apercevoir qu'il y quelqu'un qui manque : Sartre. 


\section{BIBLIOGRAPHIE}

Astruc A. \& Contat M. 1977. Sartre. Paris. Gallimard.

Beauvoir S. 1981. La cérémonie des adieux (suivi de Entretiens avec Jean-Paul Sartre aoûtseptembre 1974). Paris. Gallimard.

Buisine A. 1986. Laideurs de Sartre. Lille. Presses Universitaires de Lille. Idt G. 1976. L'autoparodie dans Les mots de Sartre. Cahiers du vingtième siècle 6. 53-86.

Idt G. 1996. Les mots de Jean-Paul Sartre. In Julien A.-Y., Maurel A., Robier M. \& Idt G. Un thème, trois cuvres, l'écriture de soi. Paris. Éditions Belin. coll. Sup Lettres. 141-199.

Lejeune Ph. 1975. L'ordre du récit dans Les Mots de Sartre. In Le pacte autobiographique. Paris. Seuil. 197-243.

Lejeune Ph. 1980. Je est un autre. Paris. Seuil.

Lejeune Ph. 1998. Les brouillons de soi. Paris. Seuil.

Sartre J.-P. 1943. L'être et le néant. Paris. Gallimard.

Sartre J.-P. 1964. Les mots. Paris. Gallimard.

Sartre J.-P. 1971-72. L'idiot de la famille. Paris. Gallimard.

Sartre J.-P. 1976. Situations X - Politique et autobiographie. Paris. Gallimard.

Sartre J.-P. 1995. Qu'est-ce que la littérature. Paris. Gallimard.

Villani J. 1996. Leçon littéraire sur Les mots de Sartre. Paris. PUF.

\section{The Words: Echoes of silence in Jean-Paul Sartre's autobiography}

ABSRTACT: The sartrean autobiographical text The Words may be read as a testament that paradoxically establishes the silence through the excessive word. Sartre says when he does not utter, silences when he speaks and, through the dialectal articulation that he establishes between uttering and silencing, offers us a truly original autobiographical form.

Keywords: Jean-Paul Sartre, autobiography, silence, word, paradox. 